\title{
TARMED veraltet? Braucht es eine Revision?
}

\author{
F. Bossard
}

Die zunehmenden Zahlen der Änderungsanträge an die PIK und PTK, die zunehmende Verunsicherung im Bereiche der schwankenden - vor allem sinkenden - Taxpunktwerte und von vielen Kollegen und Fachgesellschaften vorgebrachte Unzufriedenheit sind Anlass, auf Probleme im TARMED hinzuweisen, welche in Bälde anzugehen sind, wollen die Vertragspartner nicht Gefahr laufen, dass das System aus dem Gleichgewicht gerät. Auch für die verschiedenen Spitäler - öffentlich-rechtlich und private Spitäler - werden tarifarische Probleme zunehmend evident. Mit Taxpunktwertkorrekturen allein wird TARMED in Zukunft nicht mehr steuerbar sein.

Verschiedene kleinere und grössere Mängel wurden, sind und werden zwar laufend behoben, die Lösungen sind aber als Übersicht nur schwer zu finden.

Das Festhalten an der aktuellen Version unter der Kostenneutralität und der inzwischen vergangenen Fallkostenstabilisierung muss nicht nur im Hinblick auf eine weitere Kostenkontrolle in allen Versicherungsbereichen überlegt werden, sondern muss auch mit einer Gesamtrevision der relevanten Eckwerte und Steuergrössen, teilweise auch der Modellmechanik verbunden werden.

Die bisher erfolgreiche Überarbeitung der Dignitäten mit den Fachgesellschaften durch M. Butz, Tarifdienst FMH, ist ein erster Schritt.

\section{Eckwerte}

TARMED baut auf drei unterschiedlichen Berechnungsmodellen auf. Zwei Modelle betreffen die technische Leistung: Das KOREG-Modell geht von erhobenen, also empirischen Daten aus, gewonnen aus der rollenden Kostenstudie, während das INFRA-Modell von normativen Eckwerten ausgeht. Und diese normativen Eckwerte stehen im Vordergrund der hier eingebrachten Kritik. Das dritte Modell ist die Berechnung der ärztlichen Leistung $\mathrm{AL}$, welche völlig unabhängig ist.

Der Aufbau von TARMED wurde in der Schweizerischen Ärztezeitung 25/2001 sehr gut beschrieben [1], was aber in diesem Manuskript fehlt, ist der Hinweis aus welcher Zeit die Eckwerte in den verschiedenen Datenbanken stammen.
Diese für das ganze System essentiellen Werte wurden bis heute nie nachgeführt! Dies, obwohl immer behauptet wurde, TARMED sei dynamisch und alle Daten und Eckwerte könnten jährlich (!) nachgeführt werden. Nachfolgend ein paar Beispiele:

Die Datenbank der «Löhne Personal» stammen aus einer Erhebung von 1997, Landesindex der Konsumentenpreise und Zürcher Baukostenindex stammen aus demselben Zeitraum, die Gerätepreise und Preise der Praxisausrüstung stammen aus dem Jahre 1996 und so weiter. Viele Eckwerte im Personalwesen sind nicht oder sehr ungenügend definiert: beispielsweise wird die produktive Arbeitszeit des Personals mit umstrittenen Ansätzen berechnet. Weiter gibt es eine offene unproduktive Arbeitszeit, ferner eine Verteilzeit und auf Leistungsebene eine Wechselzeit. Zwischenzeitlich sind die 50-Stunden-Woche für die Ärzte in den Spitälern eingeführt und wesentlich gekürzte Arbeitszeiten des nichtärztlichen Personals.

Die Berechnung der Personalkosten ist an die Raumzeit gekoppelt und basiert auf zum Teil nicht korrekten normativen Eckwerten (Tagesvorbereitungszeit, Raumbelegungs- und Wechselzeit sowie indirekten Zeiten und Korrekturfaktoren).

Im weiteren sind auch die der TL zugeordneten Raumflächen erheblich umstritten, ebenso die Preise und Amortisationszeit der medizinischen Anlage- und Gerätekosten, die sogenannten Anlagenutzungskosten.

Ein erhebliches Problem im aktuellen TARMED ist der SUK-Satz, die Sach- und Umlagekosten. Dieser Eckwert kommt aus dem Spitalbereich und ist inhaltlich nirgends genau präzisiert. Der SUK-Satz beinhaltet im wesentlichen: Infrastrukturkosten, Materialkosten, Versicherungen usw.

Wenn in einer Berechnung ein Faktor, der immerhin in fast allen Sparten 65-70\% der Kosten ausmacht, einbezogen wird, sollte dessen normative Dimensionierung doch empirisch unterstützt sein; und hier genügen die umstrittenen Angaben von sieben Spitälern nicht. Ausserdem ist es befremdend, wenn alle Sparten SUK-Sätze von 65 bis 70\% aufweisen, aber nur die Sparten 186 (CT) und 187 (MRI) einen SUKSatz von $43 \%$ !
Dr. med. François Bossard Spychertenstrasse $37 \mathrm{C}$ CH-3652 Hilterfingen 
Im Rahmen des RE II Radiologie wurde eine bessere Version des SUK-Satzes entwickelt, deren Eckwerte begründbar sind und welcher auf alle übrigen Sparten übertragbar ist. Gegen solche Veränderungen wehren sich $\mathrm{H}+$ und die Versicherer.

In diesem Zusammenhang muss auch noch auf die Problematik der Materialkosten hingewiesen werden. Alle erinnern sich noch daran, als im TARMED Alpha 3.0 Materialkosten bis Fr. 200.- in den medizinischen Leistungen enthalten gewesen sind. Diese Zahl wurde nach einer stürmischen Ärztekammersitzung auf Fr. 3.- gesenkt, ohne dass dies klar im TARMEDAlgorithmus sichtbar geworden ist.

Als generelle Anmerkung zu allen Eckwerten muss folgendes aus meiner Sicht festgehalten werden: Will eine tarifarische Berechnung den Anspruch erheben, betriebswirtschaftlich zu sein, so müssen diese Eckwerte auch der Realität entsprechen und deshalb auf erhobenen Werten beruhen. Deshalb erstaunt es, dass nicht einmal die aktuellen Eckwerte der rollenden Kostenstudie in TARMED übernommen werden.

Eigentlich sollte es klar sein, dass alle zeitfixierten Eckwerte wie Löhne, Index der Sozialkosten, Baukostenindex, LIKP, aber auch Kosten aus der rollenden Kostenstudie usw. am Ende der Kostenneutralität an die Realität anzupassen wären. - Ein Grund mehr, die Revision heute schon an die Hand zu nehmen.

\section{Mengengerüst}

Eine weitere wichtige Datensammlung im TARMED sind die Häufigkeiten, also das Mengengerüst aller Leistungen. Wir haben bereits 1999 für die Radiologie herausgefunden, dass das Mengengerüst gewaltige Fehler aufwies: So wurden im TARMED 16711665 Röntgenuntersuchungen allein in Sparte 180 hinterlegt, während effektiv von allen Ärzten und allen Spitälern zusammen in der Schweiz 2409070 Untersuchungen durchgeführt wurden. Ähnlich die Situation in den anderen Sparten: Durchleuchtungen hinterlegt: 416865, effektiv durchgeführt: 132595; Mammographien hinterlegt: 2407 410, effektiv durchgeführt 216 727; CT hinterlegt: 688970 , effektiv durchgeführt 366750 ; MRI hinterlegt: 482335 , effektiv durchgeführt: 238 653. Etwas geringer sind die Abweichungen im Ultraschall und in der Angiographie.

Die Mengengerüste wären übrigens heute schon mit den elektronisch abgerechneten Leistungen überprüfbar. Dies allerdings nur für jenen Teil der freipraktizierenden Ärzte, welche über die TrustCenter abrechnen. Weder H+ noch santésuisse verfügen zurzeit über gesamtschweizerische Daten in entsprechender Differenzierung. Die Daten der MTK sind auf den Bereich UV/MV/IV beschränkt.

Interessanterweise ist das hinterlegte Mengengerüst im TARMED die erste Grundlage für die Kostenneutralität beziehungsweise die Fallkostenstabilisierung.

\section{Leistungen, Minutage und Berechnungs- modelle}

Unklar sind auch verschiedene Rechnungsvorgänge im TARMED. Wenn man die Franken pro Minute der Sparte Röntgen I aus dem Tarifbrowser ermittelt (TL-Betrag dividiert durch die TL-Minuten), so erhält man Fr. 3.90 pro Minute, während die entsprechende Tabelle im INFRAModell Fr. 4.15 pro Minute aufweist. Umgekehrt im Ultraschall, dort ermittelt man Fr. 3.32 pro Minute und in der entsprechenden Tabelle findet man Fr. 2.40 pro Minute. Dies sei angeblich auf die Differenz INFRA-Modell und KOREG-Modell zurückzuführen, aber niemand weiss genau wie und weshalb.

Merkwürdig ist auch eine andere Tatsache, welche sich bei der Analyse der Leistungen eines Internisten oder Grundversorgers ergibt: Die AL gemäss Tarifbrowser beträgt Fr. 1.91,4 pro Minute, während die TL Fr. 1.63,9 pro Minute beträgt. Und im INFRA-Modell weist das Sprechzimmer eine TL von Fr. 1.22,7 pro Minute auf. Multipliziert man die Leistungen eines Grundversorgers während eines Jahres aus, so kommt man auf ein Verhältnis AL zur TL von rund 55\% zu 45\%. Damit lässt sich zwar zeigen, dass die ärztliche Leistung höher bewertet wird, aber ob sich dies mit den Resultaten der rollenden Kostenstudie deckt, wonach die Betriebskosten bei einem Internisten oder Grundversorger 65 bis $75 \%$ betragen sollen, bezweifeln wir.

Hier wird jeweils argumentiert, dass ja der Umsatz über TARMED ja nur einen Teil des Praxisumsatzes darstelle. Der Umsatz TARMED betrage $66 \%$, 7\% des Umsatzes basiert auf Labordiagnostik, 25\% über Medikamentenabgabe und 0,7\% über Physiotherapie. Aufwandmässig wird dies wie folgt aufgeschlüsselt: TARMED-Leistungen 51\%, Vorsorge 6\%, Labor 9\%, Medikamentenabgabe $32 \%$ und Physiotherapie 1\%; die Eckwerte des Belegarztes sind in diesem Zusammengang unbekannt bzw. nicht einbezogen.

Die frühere FMS hat damals mit dem Gutachten Neubauer ausserdem auf unklare Koeffizienten und Steuergrössen im Berechnungs- 
algorithmus hingewiesen, deren Bedeutungen ebenfalls zu klären wären.

In vielen Anträgen an die PTK wird die Minutage einzelner Leistungen beanstandet, weil diese Minutage nicht der Realität entspricht. Dies betrifft nicht nur die AL-Minuten, sondern auch in vielen Fällen die TL-Minuten, welche in einzelnen Leistungen nicht identisch sind!

\section{Dignitäten}

Dass die Dignitäten bereits zu erheblichen Problemen geführt haben, konnte man nicht nur an der Ärztekammer erfahren, sondern ist auch an den Revisionsbemühungen des Tarifdienstes ersichtlich, welcher zurzeit die qualitativen Dignitäten revidiert; in einer zweiten Phase müssen sicher auch die quantitativen Elemente überarbeitet werden.

Wie und wo der Steuerungsfaktor von 1,2 zugunsten aller operativ-invasiven Leistungen gemäss Ärztekammerbeschluss vom 2. Februar 2000 in TARMED eingeflossen ist, konnten wir auch nicht nachvollziehen.

Allerdings wird die AL nicht nur durch die Dignitäten definiert, sondern durch das Referenzeinkommen und die Produktivität. Dies darf nicht mit der Auslastung verwechselt werden, welche sich auf die TL bezieht. Dabei wird die Auslastung selber nicht als Steuergrösse eingegeben; sie muss im INFRA-Modell aus verschiedenen Eckwerten mit der durchschnittlichen Untersuchungszeit ermittelt werden. Die Auslastung im KOREG-Modell ist nicht klar.

\section{Auslastung in der Radiologie und in anderen Fächern}

Und bei der Auslastung sind einige besonders realitätsfremde Annahmen zu erwähnen, die in den bildgebenden Systemen getroffen wurden: Rechnet man die Auslastung der Sparte 180, Röntgen I, aus (Betriebszeit dividiert durch durchschnittliche Untersuchungszeit), so erhält man 7796,5 Untersuchungen pro Jahr, dies ergibt 31,8 Untersuchungen pro Tag in einer Betriebsschicht von 8,4 Stunden pro Tag (welcher Praktiker macht soviel?). Ähnlich liegen die Werte in der Mammographie (4297,8 Untersuchungen pro Jahr $=17,4$ Untersuchungen pro Tag), Ultraschall (5189,5 Untersuchungen pro Jahr $=21,05$ Untersuchungen pro Tag). Kann mir jemand irgendeinen Internisten oder irgendein Spital angeben, wo 21 US-Untersuchungen pro Tag an einem Gerät in einer
Betriebsschicht durchgeführt werden? Der Ultraschallarzt müsste bei gleicher Rechnung während 10 Stunden ununterbrochen diese Ultraschalluntersuchungen abarbeiten!

Hier noch eine ähnliche Berechnung auf dem Gebiet der operativen Leistungen: Der OP II muss pro Jahr 100845 Minuten ausgelastet sein. Eine offene Cholezystektomie (20.2670) dauert 30 Minuten; somit müssten 3362 solche Operationen pro Jahr bzw. 13,5 solche Operationen pro Tag in einem Operationssaal durchgeführt werden. Wird eine Laparatomie (20.0620) vorgeschaltet, kommen 56 Minuten hinzu, somit 1173 Operationen pro Jahr entsprechend 4,7 Operationen pro Tag.

Bleiben wir bei den Beispielen der Auslastung und schauen wir uns den Hausarzt an. Sein Sprechzimmer hat eine jährliche Betriebszeit von 132500 Minuten. Bei einer durchschnittlichen Konsultationsdauer von 15 Minuten ergibt dies 8833 Patienten pro Jahr oder 35,3 Patienten pro Tag!

Es ist für uns kaum möglich, in allen Fachbereichen mit gleicher Akribie merkwürdige Eckwerte zu eruieren (auch wenn ich bereits einige gefunden habe), aber ausgehend von der Systematik im TARMED kann ohne negative Spekulation gesagt werden, dass auch an anderen Orten von TARMED, in anderen Fachbereichen und Leistungskategorien erhebliche Mängel vorhanden sind, welche nun als Verzerrungen in den Auswertungen der Assessmentkommission und teilweise auch schon in Kostenneutralitätsbüro zum Vorschein kommen.

\section{Kostenneutralität und Fallkosten- stabilisierung}

Die Elemente Kostenneutralität und Fallkostenstabilisierung sind nachträglich und eigentlich nahezu unabhängig von KOREG und INFRAModell in den Komplex der TARMED-Verträge hineingeflossen.

Für die Berechnung der kostenneutralen Starttaxpunktwerte scheinen auch die Mengengerüste und die Transcodierung eine grosse Rolle zu spielen. Die Transcodierung heisst die «Übersetzung» der bisherigen kantonalen Tarife in TARMED, also die Zuordnung der Leistungen nach kantonalen Tarifen zu den Leistungen im TARMED; dies ging naturgemäss nicht überall einfach und ist eine weitere Fehlerquelle.

Die Diskussion dieser beiden Varianten wären eine eigene Abhandlung wert. Es sei hier nur festgehalten, dass nun erste Erfahrungen vorliegen und damit Punkte offensichtlich wer- 
den, welche in dieser Form nicht in zukünftige Verträge einfliessen dürfen. Dass es weiterer Verträge bedarf, ist nur schon wegen der Kostenentwicklung und des Teuerungsausgleiches notwendig,

Vor allem die Diskussion um die gewollte Aufwertung der ärztlichen Leistungen gegenüber den technischen Leistungen gibt im Spiegel der nun wirklich überschiessenden Grundleistungen zu intensiven Diskussionen Anlass.

\section{PIK, PTK, FAQ}

Die vielen insgesamt über hundert PIK- und PTK-Entscheide sowie die unzähligen FAQs sollten in einer neuen revidierten TARMEDFassung ebenfalls nachgeführt werden und in den Tarifbrowser eingearbeitet werden.

Die Nachführung ist notwendig, weil einzelne PIK- und PTK-Entscheide nicht mehr notwendig oder nicht mehr aktuell sind.

Verschiedene FAQs sind anzupassen. Die Koordination mit den FAQs der Vertragspartner ist anzustreben sowie der Einbezug der Softwarefirmen.

Alle positionsweisen Anmerkungen können sehr gut bei den einzelnen Positionen eingefügt werden, während für die übrigen Anmerkungen diese in einem Anhang eingebracht werden können.

Ferner ist zu erörtern, inwieweit auch Fragen der PaKoDig in den Browser einzuarbeiten sind, ferner die Merkblätter bezüglich Rechnungsformular, Diagnosecode usw.

\section{Software, XML-Format, Validator}

Weitere Problemkreise sind die elektronische Übertragung der Rechnungsdaten, die elektronische Prüfung der Rechnungsdaten mit Validator, der Validator selber und zuletzt die elektronische Überprüfung der Dignität der Rechnungssteller. Allein diese Punkte wären eine Abhandlung wert.

\section{Perspektiven und Schlussfolgerung}

Meine Aufzählung der Mängel ist sicher unvollständig. Sie berührt nur einige wesentliche Kernelemente, aber nicht alle Fehlerquellen, welche zu den aktuellen Unwuchten geführt haben.

Die unzähligen Korrekturfaktoren in den Datenbanken von KOREG und INFRA sind glei- chermassen zu überprüfen wie alle oben genannten Eckwerte. Auch das Zusammenführen der beiden Modelle mit offener Berechnungsstruktur und Eckwerten ist notwendig, um das Vertrauen in das Tarifwerk zu fördern.

TARMED reloaded darf nicht täuschen: damit ist keine Revision beabsichtigt; der Berechnungsalgorithmus von damals mit alten Accessversionen ist schwerfällig. Deshalb ist eine neue Version notwendig, und zudem in Händen einer neutralen Organisation.

Während TARMED in den neunziger Jahren in seinen Auswirkungen schwierig prognostizierbar war, ist heute mit der digitalen und elektronischen Abrechnung nicht nur die Tarifstruktur kontrollierbar, sondern auch das Abrechnungsverhalten der Leistungserbringer. Damit sollten evidente Unwuchten und Verzerrungen im TARMED besser korrigiert werden können.

Deshalb erscheint es uns dringend angezeigt, TARMED einer Gesamtrevision unter Einbezug aller Fachgesellschaften zu unterziehen. Dabei müssen die Gegebenheiten aller Leistungserbringer korrekt einbezogen werden, so dass TARMED den Namen eines betriebswirtschaftlich berechneten Tarifes wirklich verdient. Aktuell ist dies nicht der Fall. Auch sollte ein gesamtschweizerischer Tarif, der sich immerhin auf mehrere Milliarden Franken Gesundheitskosten auswirkt, sorgfältiger abgestützt sein und Korrekturmöglichkeiten voll in Anspruch nehmen. Die Vertragspartner sollten den Aufwand nicht scheuen.

Da immerhin fast die Hälfte der Mitglieder der FMH im Spitalbereich tätig sind, ist die tarifarische Zusammenarbeit mit $\mathrm{H}+\mathrm{zu}$ intensivieren oder die Führung zu übernehmen.

TARMED birgt es in seiner fachspezifischen Gliederung in sich, dass ohne relevante FMHinterne Grabenkämpfe eine Revision durchgezogen werden könnte. Und ausserdem ist mit der Revision der Dignitäten eine sehr heikle Aufgabe bereits geschafft.

Elemente wie Teuerung, demographische Verschiebungen, zunehmende Überalterung, Fortschritte der Medizin in Diagnostik und Therapie usw. sollten im Rahmen der Kostenneutralität oder genereller gesagt einer Kostenkontrolle herausgenommen werden. Hier sind auch die Auswirkungen von «Nebenkosten» wie Labor, Arzneimittel, Physiotherapie usw. korrekt abzugrenzen. Lehre und Forschung sind ja bereits generell aus TARMED ausgeklammert.

Die Berücksichtigung politischer Vorgaben muss aus dem Modell herausgenommen und klar getrennt ausgewiesen und verhandelt werden. 
Die Motivation zu einer solchen Revision dürfte wegen des Arbeitsumfanges generell nicht sehr gross sein, FMH-intern wie auch bei den Vertragspartnern. Ich bin aber der Auffassung, dass die FMH dazu die Führung übernehmen muss und ihre Arbeit breit abstützen soll.

Der Arbeitsaufwand einer solchen Revision ist nicht zu unterschätzen, darum wären Vorbereitungen bereits jetzt einzuleiten. Da diese Arbeit vor allem fachbereichsorientiert ist, sind die Fachgesellschaften unter einer kleinen Stabsgruppe einzubinden.

Die Vorbereitungsarbeiten und der Aufwand zu einer Revision sind auch auf seiten der Ver- sicherer nicht zu unterschätzen. Deshalb kann eine solche Revision bereits vor Ende der Kostenneutralität Sommer 2005 beginnen, denn sie wird kaum bis zu diesem Zeitpunkt abgeschlossen sein; andererseits kann eine neue Phase der Kostenkontrolle auf den Beginn der revidierten TARMED-Fassung hinausgeschoben werden.

\section{Literatur}

1 Arbeitsgruppe TarMed FMH. Die TARMED-BlackBox, die keine Black-Box ist. Schweiz Ärztezeitung 2001;82(25):1312-4. 